\title{
ARTíCULO \\ Cambios interanuales en la talla de madurez de sardina común, Strangomera bentincki, en la zona centro-sur de Chile (2007-2012)
}

Interannual changes in size-at-maturity of common sardine, Strangomera bentincki, in the central-southern zone of Chile (2007-2012)

\section{Blanca Bustos ${ }^{1 *}$ and Luis A. Cubillos ${ }^{1,2^{* *}}$}

\begin{abstract}
'COPAS Sur-Austral, Universidad de Concepción, Casilla 160-C, Concepción, Chile.*blancabustos@udec.cl,**lucubillos@udec.cl ${ }^{2}$ Departamento de Oceanografía, Facultad de Ciencias Naturales y Oceanográficas, Universidad de Concepción, Casilla 160-C, Concepción, Chile
\end{abstract}

\begin{abstract}
Interannual changes in size-at-maturity $\left(\mathrm{L}_{m}\right)$ of common sardine, Strangomera bentincki, were evaluated for the period 2007-2012 in the south-central area of Chile. Data consisted of microscopic maturity stages of female ovaries, which were collected during the reproductive peak each year. Immature females were identified from mature females and a logistic model was fitted to data through generalized linear model (GLM) and generalized linear mixed model (GLMM). The best model was characterized by having both variable slope and intercept, revealing significant interannual variations in size-at-maturity. Although the average length of the immature fraction showed a tendency to decline over time, interannual changes in size-at-maturity varied at random between 8.4 and $12.7 \mathrm{~cm}$, and around $\mathrm{L}_{\mathrm{m}}=10.7 \mathrm{~cm}$ total length. It is concluded that GLMM technique was suited to representing interannual random changes in size-at-maturity of common sardine. There was no trend to diminish according to the hypothesis of truncation of size/age due to fishing intensity. Rather, the random expression in size-at-maturity could be a consequence of factors associated with sampling, growth or delayed response in maturity due to spatio-temporal asynchrony in the reproductive process.
\end{abstract}

Key words: Reproductive parameter, size at maturity, common sardine, small pelagic fish, pelagic fisheries

Resumen.- Se evaluaron cambios interanuales en la talla de madurez $\left(L_{m}\right)$ de sardina común, Strangomera bentincki, para el periodo 2007-2012 en la zona centro-sur de Chile. Los datos consistieron en estados de madurez microscópicos de los ovarios de las hembras que fueron colectadas durante la época de máxima actividad reproductiva en cada año. Se identificaron las hembras maduras de las inmaduras, y se ajustó el modelo logístico de madurez utilizando modelos lineales generalizados (MLG) asumiendo independencia entre años, y modelos lineales generalizados de efectos mixtos (MLGM) asumiendo efectos aleatorios asociados a los años. El mejor modelo se caracterizó por tener intercepto y pendiente variable, revelando cambios interanuales significativos en la talla de madurez. Aunque en los años más recientes se observó una tendencia leve a disminuir en la talla media de la fracción inmadura, la talla de madurez fluctúo aleatoriamente entre 8,4 y $12,7 \mathrm{~cm}$, y alrededor de $\mathrm{L}_{\mathrm{m}}=10,7 \mathrm{~cm}$ de longitud total. De esta manera, se concluye que el MLGM es más adecuado para representar cambios interanuales aleatorios en la talla de madurez de la sardina común, ya que no hubo una tendencia a la disminución que pudo ser atribuible a la intensidad de pesca bajo la hipótesis de truncamiento de la estructura de tallas/edad. Más bien, en la expresión de los cambios aleatorios en la talla de madurez pueden ser consecuencia de factores asociados ya sea al muestreo, al crecimiento o a los retrasos en la madurez debido a la asincronía espacio-temporal del proceso reproductivo.

Palabras clave: Parámetro reproductivo, talla de madurez, sardina común, pez pelágico pequeño, pesquería pelágica

\section{INTRODUCCIÓN}

La sardina común Strangomera bentincki (Norman, 1936), es un pez pelágico endémico de Chile, cuya distribución se extiende desde Coquimbo $\left(29^{\circ} 95^{\prime} \mathrm{S} ; 71^{\circ} 35^{\prime} \mathrm{W}\right)$ hasta la Península de Taitao (46 $36^{\circ} \mathrm{S} ; 7^{\circ}{ }^{\circ} 25^{\prime} \mathrm{W}$ ) (Vásquez et al. 2010), y habita preferentemente en zonas cercanas a la costa, donde la productividad biológica es generalmente alta debido a eventos de surgencia que en la zona centro-sur de Chile se presentan con mayor frecuencia entre septiembre y marzo
(Arcos 1987). S. bentincki se caracteriza porque su tamaño no supera $20 \mathrm{~cm}$ de longitud total, por tener un ciclo de vida corto ( $<5$ años de edad), cambios estacionales en la tasa de crecimiento, y una tasa de mortalidad natural alta (Cubillos et al. 1999, 2002).

En los aspectos reproductivos, S. bentincki es un pez iteróparo, con fecundidad indeterminada, y alcanza la primera madurez a un tamaño de entre 10 y $11 \mathrm{~cm}$ de longitud total, al 
finalizar el primer año de vida (Arrizaga 1981, Mujica \& Rojas 1984, Arancibia et al. 1994, Cubillos et al. 1999). La actividad reproductiva se extiende desde julio a octubre, con un máximo entre agosto y septiembre (Cubillos et al. 1999, 2001; Cubillos $\&$ Arcos 2002, Claramunt et al. 2014).

Además, $S$. bentincki es un recurso de importancia para una flota artesanal e industrial de cerco que opera en la zona centro-sur de Chile ( $\left.33^{\circ} 00^{\prime} S ; 41^{\circ} 30^{\prime} \mathrm{S}\right)$, con Talcahuano ( $36^{\circ} 44^{\prime} \mathrm{S} ; 73^{\circ} 06^{\prime} \mathrm{W}$ ) como el principal puerto de desembarque (Cubillos et al. 1998). Los desembarques de S. bentincki han fluctuado entre 281.382 y 887.272 ton entre 2000 y 2014 (SERNAPESCA 2013), y son obtenidos por la flota desde marzo a junio de cada año. Por lo general, las capturas de la flota están constituidas en más del $80 \%$ por peces juveniles menores de $10 \mathrm{~cm}$ de longitud total; los cuales han reclutado a la zonas de pesca durante el primer semestre (Cubillos et al. 2002, Gatica et al. 2007, Zuñiga \& Canales 2014'). Debido a que la intensidad de pesca que se ejerce sobre los juveniles ha aumentado en los años más recientes (2010-2012), es probable que esté ocurriendo sobrepesca por crecimiento (Cubillos et al. 2014); es decir, cuando la intensidad de pesca es alta y concentrada sobre juveniles inmaduros antes que logren su máximo incremento en peso. Las consecuencias de este modo de explotación podrían producir una disminución de la supervivencia de los juveniles, y disminuir la probabilidad que una fracción importante llegue a reproducirse por primera vez. En este contexto, cuando la pesca es intensa y concentrada sobre la fracción juvenil, puede ocurrir lo que se llama el truncamiento de la estructura de tallas/edad al remover los ejemplares de crecimiento más alto (e.g., Anderson et al. 2008), lo que podría alterar el ciclo reproductivo estacional (Cubillos et al. 2014). Conover \& Munch (2002) concluyeron que cuando los juveniles son capturados en forma intensa, la respuesta poblacional es la selección de individuos con más alto potencial reproductivo. En este contexto, la explotación excesiva sobre juveniles podría inducir cambios en la talla de madurez, particularmente entre 2007 y 2012; periodo en que se ha registrado una intensidad de pesca mayor sobre la fracción juvenil del stock. En este contexto, el objetivo de este estudio fue evaluar cambios interanuales significativos en la talla de madurez de $S$. bentincki en la zona centro-sur de Chile.

\section{MATERIALES Y MÉTODOS}

\section{Fuente de DATOS E INFORMACIÓN}

Se utilizó la base de datos de las hembras de $S$. bentincki que fueron colectadas en los cruceros del Método de la Producción Diaria de Huevos (MPDH) realizados entre los años 2007 a 2012 en la zona centro-sur de Chile (Fig. 1), (Cubillos et al. 2007, Cubillos et al. 2013). En estos cruceros, el diseño

Figura 1. Área de estudio que fue cubierta durante la aplicación del crucero del Método de Producción Diaria de huevos de sardina común en el período 2007 al 2012 /

Study area covered during the application of the Daily Egg Production survey of the sardine in 2007 to 2012 period

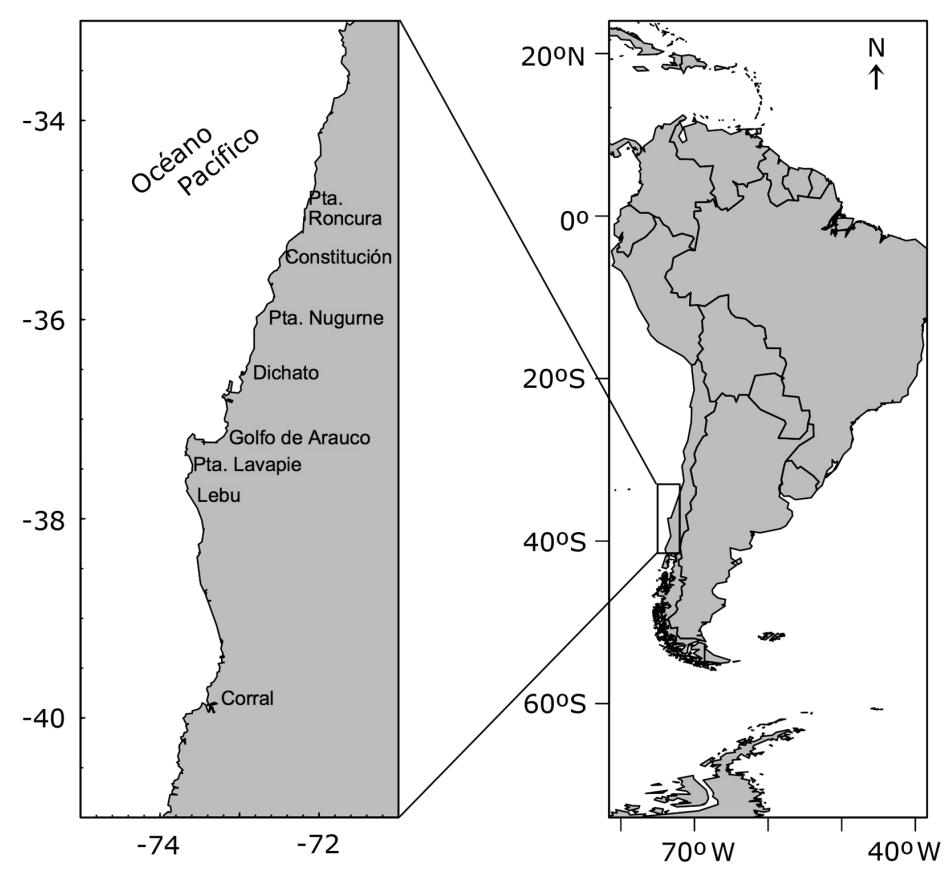

${ }^{1}$ Zuñiga MJ \& C Canales. 2014. Investigación del estatus y posibilidades de explotación biológicamente sustentables en sardina común zona centro-sur, año 2014, 153 pp. Instituto de Fomento Pesquero (IFOP), Valparaíso. 
muestral corresponde a un muestreo bi-etapico de ejemplares adultos (Cochrane 1980, Cubillos et al. 2013). Los cardúmenes se segregan según la condición reproductiva (Blaxter \& Hunter 1982), y por lo tanto las unidades de primera etapa corresponde a un número fijo de lances de pesca $(n=50)$ con el objeto de muestrear diferentes cardúmenes, y un número fijo de hembras por lance $(m=35)$ como segunda etapa. Sin embargo, el número de lances efectivos que han sido obtenidos han fluctuado entre $\mathrm{n}=30$ (2008) y $\mathrm{n}=52$ (2010), y provienen principalmente de dos sectores; desde la Bahía Concepción hasta el Golfo de Arauco y desde Puerto Saavedra hasta Corral. Por otra parte, y a diferencia de los objetivos del MPDH, en este estudio se asume homogeneidad en la unidad de stock. Asimismo, en este estudio no se descartaron los lances de pesca con ejemplares inmaduros, razón por la cual el número total de hembras que aquí se analiza fue mayor que aquellos informados en los cruceros MPDH por Cubillos et al. (2013). El número final de placas histológicas fueron de 3970 hembras en 2007, 1375 en 2008, 3450 en 2009, 2870 en 2010, 1760 en 2011 y 2526 hembras en 2012 (Tabla 1). El muestreo consistió en obtener 35 hembras de $S$. bentincki de cada lance de pesca, las cuales fueron fijadas en formalina para el análisis histológico posterior en el laboratorio. Para la fijación, las hembras fueron diseccionadas ventralmente y se conservaron en una solución de formalina al $10 \%$ tamponada. Cada hembra preservada fue medida en su longitud total $(\mathrm{cm})$, peso total $(\mathrm{g})$ y peso corporal libre de gónada $(\mathrm{g})$. Los ovarios fueron pesados $( \pm 0,01 \mathrm{~g})$ y se preservaron en solución de formalina tamponada al $10 \%$. Posteriormente, cada ovario fue cortado y teñido con hematoxilina y eosina con el objeto de lograr la preparación histológica.

El desarrollo y la maduración de los ovocitos fue dividido en 9 estados de madurez de acuerdo con las características descritas por Wallace \& Selman (1981), Selman \& Wallace (1989) y Hunter \& Macewicz (1985). De los 9 estados se caracterizaron como inmaduros a los estados de madurez sexual microscópicos (EMM) I (virginal), II (inmaduros) y IX (en recuperación); y maduros a los EMM III (en vitelación), IV (vitelado), V (en hidratación, núcleo migrante), VI (hidratado), VII-VIII (desovado, presencia de folículos postovulatorios). Si bien el estado IX corresponde a individuos desovados, no se incluyeron como maduros debido a que predominan en el ovocitos inmaduros y atresia folicular, y por lo tanto no forman parte de las hembras activas a diferencia de los post-desovante de tanda VII-VIII en que coexisten ovocitos hidratados con foliculos postovulatorios (Brown-Peterson et al. 2011).

Tabla 1. Número de hembras inmaduras (Inm) y maduras (Mad) de sardina común por año y clases de longitud de $1 \mathrm{~cm}$ de longitud total / Number of immature ( $(\mathrm{nm})$ and mature (Mad) females of common sardine by year and length-classes of $1 \mathrm{~cm}$ total length

\begin{tabular}{|c|c|c|c|c|c|c|c|c|c|c|c|c|}
\hline \multirow{2}{*}{$\begin{array}{c}\mathrm{LT} \\
(\mathrm{cm})\end{array}$} & \multicolumn{2}{|c|}{2007} & \multicolumn{2}{|c|}{2008} & \multicolumn{2}{|c|}{2009} & \multicolumn{2}{|c|}{2010} & \multicolumn{2}{|c|}{2011} & \multicolumn{2}{|c|}{2012} \\
\hline & Inm & Mad & Inm & Mad & Inm & Mad & Inm & Mad & Inm & Mad & Inm & Mad \\
\hline 7 & 0 & 0 & 0 & 0 & 0 & 0 & 0 & 0 & 0 & 0 & 2 & 0 \\
\hline 8 & 0 & 0 & 9 & 1 & 14 & 1 & 7 & 1 & 46 & 1 & 48 & 2 \\
\hline 9 & 0 & 3 & 177 & 3 & 69 & 37 & 27 & 21 & 286 & 6 & 141 & 4 \\
\hline 10 & 0 & 41 & 557 & 16 & 304 & 226 & 106 & 182 & 269 & 40 & 183 & 89 \\
\hline 11 & 23 & 85 & 154 & 25 & 173 & 290 & 66 & 149 & 36 & 41 & 44 & 185 \\
\hline 12 & 161 & 348 & 37 & 18 & 146 & 643 & 41 & 374 & 7 & 193 & 13 & 555 \\
\hline 13 & 104 & 770 & 13 & 25 & 41 & 433 & 31 & 544 & 4 & 245 & 1 & 457 \\
\hline 14 & 33 & 927 & 13 & 83 & 10 & 286 & 80 & 777 & 0 & 424 & 0 & 514 \\
\hline 15 & 14 & 689 & 12 & 142 & 6 & 296 & 32 & 325 & 0 & 122 & 0 & 194 \\
\hline 16 & 5 & 643 & 2 & 52 & 2 & 320 & 18 & 79 & 0 & 36 & 0 & 89 \\
\hline 17 & 1 & 115 & 0 & 26 & 2 & 115 & 0 & 10 & 0 & 4 & 0 & 5 \\
\hline 18 & 0 & 8 & 1 & 12 & 1 & 35 & 0 & 0 & 0 & 0 & 0 & 0 \\
\hline $\mathrm{n}$ & 341 & 3629 & 975 & 403 & 768 & 2682 & 408 & 2462 & 648 & 1112 & 432 & 2094 \\
\hline Promedio & 12,6 & 14,1 & 10,2 & 14,4 & 10,8 & 13,2 & 11,9 & 13,2 & 9,5 & 13,3 & 9,6 & 13,0 \\
\hline SD & 0,98 & 1,45 & 1,11 & 1,81 & 1,29 & 2,04 & 2,05 & 1,52 & 0,75 & 1,32 & 0,91 & 1,41 \\
\hline
\end{tabular}




\section{Análisis estadísticos}

La clasificación de la muestra total de estadios de madurez microscópica en ejemplares inmaduros y maduros sigue una distribución de probabilidad binomial, cuya proporción de maduros en función del tamaño siguen un modelo logístico, expresado por:

$$
\begin{gathered}
P=\frac{1}{1+e^{(-(\alpha+\beta * L))}} \\
L_{m}=\frac{\alpha}{\beta}
\end{gathered}
$$

donde $P$ es la proporción de hembras maduras, $L$ es la longitud total $(\mathrm{cm}), \alpha$ y $\beta$ son los parámetros del intercepto y la pendiente. La talla de madurez $\left(L_{m}\right)$ se estima a partir del cociente entre $\alpha$ y $\beta$ (Ecuación 2).

Con el objeto de estimar la talla de madurez sin efectos interanuales, la función de madurez (Ecuación 1) se ajustó combinando los datos de todos los años mediante Modelo Lineal Generalizado(MLG), con familia binomial y enlace 'logit'. Para ello, se utilizó el paquete 'MASS' de Venables \& Ripley (2002) para el programa R (R Core Team 2014).

La evaluación de los cambios interanuales en la talla de madurez se realizó considerando dos técnicas estadísticas. La primera técnica utiliza MLG asumiendo independencia entre los años, como si provinieran de poblaciones estadísticas distintas. La segunda técnica corresponde a un Modelo Lineal Generalizado de efectos Mixtos (MLGM), se asume que interanualmente ocurren efectos aleatorios que se expresan a partir de un modelo fijo de madurez en función del tamaño (Pinheiro \& Bates 2000).

La aplicación de MLGutilizó una familia binomial y enlace logit, utilizando el año como factor. El enlace logit permite relacionar la variable dependiente con las variables independientes a través del predictor lineal. Se probaron los siguientes modelos:

Modelo 1: Pendiente fija, e intercepto variable

$$
\log \left(\frac{P_{i, j}}{1-P_{i, j}}\right)=\alpha+\beta \times L_{i, j}+Y_{j}+\varepsilon_{i, j}
$$

Modelo 2: Intercepto fijo, y pendiente variable:

$$
\log \left(\frac{P_{i, j}}{1-P_{i, j}}\right)=\alpha+\beta \times L_{i, j} \times Y_{j}+\varepsilon_{i, j}
$$

Modelo 3: Intercepto y pendiente variable:

$$
\log \left(\frac{P_{i, j}}{1-P_{i, j}}\right)=\alpha+\beta \times L_{i, j} \times Y_{j}+Y_{j}+\varepsilon_{i, j}
$$

donde $P_{i, j}$ es la proporción de hembras maduras respecto del total, $\alpha$ es el intercepto, $\beta$ es la pendiente, $L_{i, j}$ es la longitud de la hembra $i$ en el año $j$, e $Y_{j}$ es el factor año. Los subíndices i y $\mathrm{j}$ representan las observaciones de longitud y de años, y $\varepsilon_{i, j}$ $\sim N\left(0, \sigma^{2}\right)$ es el error aleatorio del predictor lineal. Se utilizó el análisis de devianza para probar cambios significativos asociados con el factor año.

En el caso de MLGM, el año fue considerado como un factor aleatorio asumiendo que dichos cambios son la expresión provenientes de un modelo poblacional en que la talla de madurez es fija. Se evaluaron los siguientes modelos

Modelo 4: Pendiente fija e intercepto aleatorio

$$
\log \left(\frac{P_{i, j}}{1-P_{i, j}}\right)=\alpha+\beta \times L_{i, j}+u_{j}+\varepsilon_{i, j}
$$

Modelo 5: Intercepto fijo y pendiente aleatoria

$$
\log \left(\frac{P_{i, j}}{1-P_{i, j}}\right)=\alpha+\left(\beta+v_{j}\right) \times L_{i, j}+\varepsilon_{i, j}
$$

Modelo 6: Intercepto y pendiente aleatoria

$$
\log \left(\frac{P_{i, j}}{1-P_{i, j}}\right)=\alpha+\left(\beta+v_{j}\right) \times L_{i, j}+u_{j}+\varepsilon_{i, j}
$$

donde $\alpha$ es el intercepto fijo, $\beta$ es la pendiente fija, $u_{j}$ son los efectos aleatorios del intercepto asociados a los años $j, u_{j} \sim N(0$, $\left.\sigma_{\mathrm{u}}^{2}\right) ; v_{j}$ son los efectos aleatorios de la pendiente asociados al año $j, v_{j} \sim N\left(0, \sigma^{2}\right) ; \mathrm{y} \varepsilon_{i, j}$ representa el error aleatorio $\varepsilon_{i, j} \sim N(0$, $\left.\sigma^{2}\right)$. En los MLGM se agrega una estructura de varianza dada por el ruido aleatorio interanual; i.e., $u_{j} \sim N\left(0, \sigma^{2}{ }_{u}\right)$ y $v_{j} \sim N(0$, $\sigma_{\mathrm{v}}^{2}$ ), la cual se asumió homogénea y sin estructura (Pinheiro $\&$ Bates 2000). Para el ajuste de MLGM se utilizó el paquete 'Ime4' de Bates (2010) para el programa R (R Core Team 2014).

El mejor modelo fue seleccionado con el Criterio de Información de Akaike (AIC). Este estadístico se basa en el valor de la verosimilitud y el número de parámetros involucrados en el modelo. El valor más bajo del AIC se utiliza para seleccionar el modelo con más parsimonia (Akaike 1987, Buckland et al. 1997).

\section{Resultados}

Se contó con 36.312 hembras entre el 2007 al 2012, de las cuales 16.023 fueron inmaduras y 20.289 maduras (Tabla 1). Se destaca que la talla de los ejemplares inmaduros comenzó a ser más pequeña el 2011 y 2012, tal como se registra en la frecuencia de ocurrencia y longitud promedio de dicha fracción (Tabla 1). En cambio, en la talla media de la fracción madura 
se observó una menor variación durante el periodo de estudio, observándose una reducción de 14,4 cm en 2008 a 13 cm en 2012 (Tabla 1).

La proporción de hembras maduras e inmaduras se muestra por clases de talla de $1 \mathrm{~cm}$ en la Figura 2. En 2007 se observó $100 \%$ de ejemplares maduros en los 9 y $10 \mathrm{~cm}$, pero el número de ejemplares fue bajo. En 2008 la mayor proporción de individuos inmaduros se registró entre los 8 y $10 \mathrm{~cm}$, para posteriormente registrarse un aumento lento en la proporción de hembras maduras con la talla. En 2009, la proporción de hembras maduras fue cercana al $50 \%$ a los $10 \mathrm{~cm}$. La proporción de hembras maduras fue similar entre el $2010 \mathrm{y}$ 2012. El 2011 se observó una baja proporción de hembras maduras entre los 8 y $10 \mathrm{~cm}$. En 2012, la proporción de hembras maduras aumentó a partir de los $10 \mathrm{~cm}$ (Fig. 2). Asimismo, en la Figura 2 se muestra el ajuste de un modelo lineal generalizado (MLG) a la totalidad de los datos, lo que resultó en una talla de madurez de 10,7 cm de longitud total, con un ajuste significativo. Los parámetros de este modelo fueron: $\alpha=-11,1$ (error estándar=0,1996, $P<0,05$ ) y $\beta=1,034$ (error estándar $=0,0174, P<0,05$ ), con una devianza residual (= 10365) significativa $(P<0,05$, test chi-cuadrado) respecto de la devianza nula (=16968) para 15952 grados de libertad.

La evaluación de cambios interanuales con MLG, mostró que el factor año fue significativo en todos los modelos $(P<$
0,05). Sin embargo, según el criterio de información de Akaike (AIC), el mejor ajuste con modelo lineal generalizado (MLG) fue el Modelo 3, con intercepto y pendiente variable (AIC= $8731,2)$, comparado con el Modelo $1(\mathrm{AIC}=9541,9)$ y Modelo $2(\mathrm{AIC}=9570,3)$. Los resultados del ajuste del modelo seleccionado se muestran en la Tabla 2, y los cambios interanuales en la talla de madurez en la Tabla 3, observándose fluctuaciones entre 8,3 y 12,7 cm en el periodo de estudio. Estos cambios interanuales no mostraron una tendencia definida hacia la disminución o al aumento, los que podrían quedar mejor representados al tratar los años como un efecto aleatorio más que efectos independientes.

En este contexto, la evaluación del ajuste de los modelos lineales generalizados de efecto mixto (MLGM) mostró que el mejor modelo correspondió al Modelo 6, también con intercepto y pendiente variables (Tabla 4). El modelo 6 logró el mejor desempeño con $\mathrm{AIC}=8791,6$, comparado con $\mathrm{AIC}=$ 9571,4 del Modelo 4 y AIC $=9599,5$ del Modelo 5. Los efectos fijos del Modelo 6 permitieron estimar la talla de madurez en 10,7 cm LT (Tabla 4), con cambios aleatorios interanuales que fluctuaron entre $8,4 \mathrm{~cm}$ en el 2010 y $12,7 \mathrm{~cm}$ LT en el 2008 (Tabla 4). La varianza del efecto aleatorio asociada al intercepto fue $\sigma^{2}=69,24$ y la varianza de los efectos aleatorios asociados a la pendiente fue $\sigma_{\mathrm{v}}^{2}=0,579$.

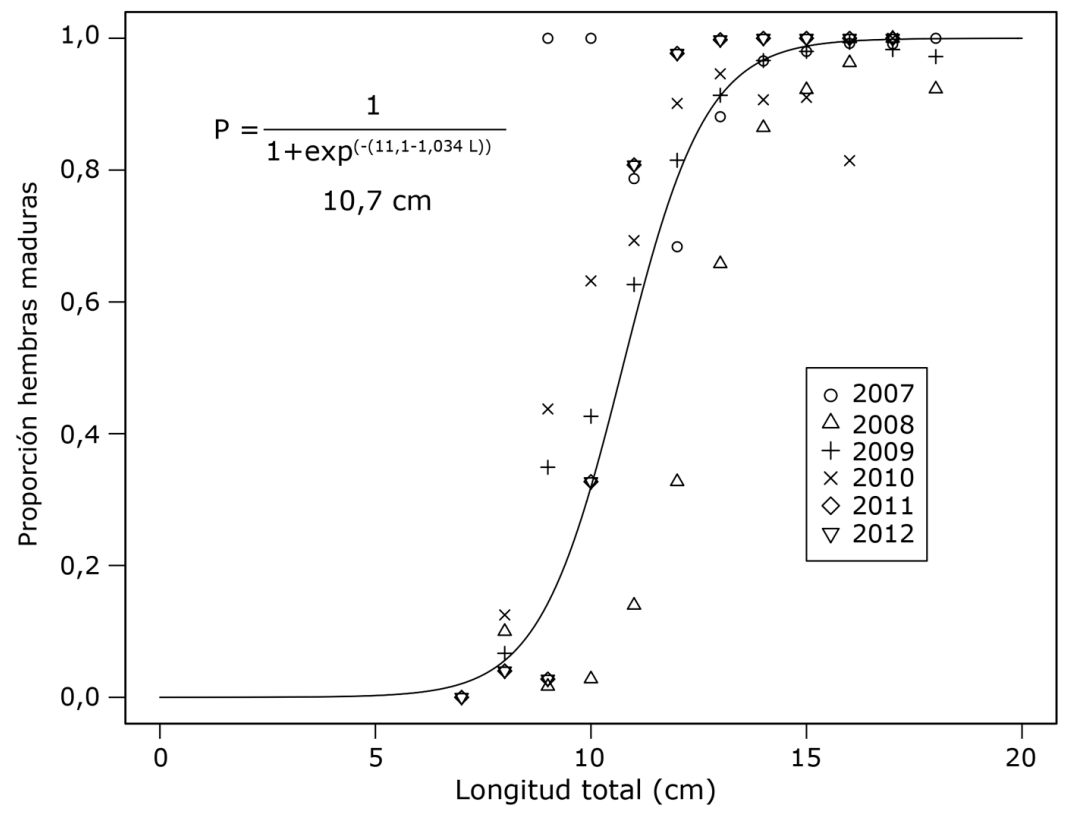

Figura 2. Proporción de hem bras maduras en función de la longitud total, sin considerar cambios interanuales en la madurez

/ Mature female proportion as a function of total length, without taking into account interannual changes in maturity 
Tabla 2. Coeficientes estimados del Modelo 3, con intercepto y pendiente variable utilizando modelo lineal generalizado (MLG), familia binomial y enlace logit en el periodo 2007-2012 (AIC=8731,2) / Coefficients estimated for Model 3 , with intercept and slope variable by using generalized (GLM), family binomial and link logit in the period 2007-2012 (AIC= 8731.2)

\begin{tabular}{lrrrr}
\hline Coeficientes & Estimación & $\begin{array}{c}\text { Error } \\
\text { Estándar }\end{array}$ & Valor Z & $\operatorname{Pr}(>|Z|)$ \\
\hline Intercepto & $-7,759$ & 0,601 & $-12,909$ & $<0,05$ \\
Pendiente & 0,758 & 0,047 & 16,276 & $<0,05$ \\
2008 & $-7,448$ & 0,937 & $-7,947$ & $<0,05$ \\
2009 & $-1,183$ & 0,733 & $-1,613$ & 0,107 \\
2010 & 4,246 & 0,720 & 5,899 & $<0,05$ \\
2011 & $-16,972$ & 1,481 & $-11,461$ & $<0,05$ \\
2012 & $-17,598$ & 1,485 & $-11,851$ & $<0,05$ \\
L*2008 & 0,443 & 0,075 & 5,857 & $<0,05$ \\
L*2009 & 0,107 & 0,060 & 1,808 & 0,071 \\
L*2010 & $-0,335$ & 0,057 & $-5,928$ & $<0,05$ \\
L*2011 & 1,525 & 0,135 & 11,295 & $<0,05$ \\
L*2012 & 1,678 & 0,136 & 12,339 & $<0,05$ \\
\hline
\end{tabular}

Tabla 3. Cambiosinteranuales en la talla de madurez de sardina común asumiendo el año como factor independiente con modelo lineal generalizado (MLG), y asumiendo el año como un efecto aleatorio con modelo lineal generalizado de efecto mixto (MLGM) / Interanual changes in the size at maturity of the common sardine considering year as an independent factor with generalized linear model (GLM), and considering year as a random effect with mixed-effect generalized linear model (GLMM)

\begin{tabular}{ccc}
\hline Año & $\begin{array}{c}\text { MLG } \\
\mathrm{L}_{\mathrm{m}}(\mathrm{cm})\end{array}$ & $\begin{array}{c}\text { MLGM } \\
\mathrm{L}_{\mathrm{m}}(\mathrm{cm})\end{array}$ \\
\hline 2007 & 10,2 & 10,3 \\
2008 & 12,7 & 12,7 \\
2009 & 10,3 & 10,3 \\
2010 & 8,3 & 8,4 \\
2011 & 10,8 & 10,8 \\
2012 & 10,4 & 10,4 \\
\hline
\end{tabular}

Tabla 4. Estimaciones de los efectos fijos para el Modelo 6 con intercepto y pendiente aleatorios utilizando Modelo Lineal Generalizado de efecto Mixto (MLGM) en el periodo 2007-2012 (AIC $=8791,6) /$ Fixed coefficients estimated for Model 6 with random intercept and slope by using Generalized Linear Model of Mixed effects (GLMM) in the period 2007-2012 (AIC= 8791.6)

\begin{tabular}{ccccc}
\hline Coeficientes & Estimación & $\begin{array}{c}\text { Error } \\
\text { Estándar }\end{array}$ & Valor Z & $\operatorname{Pr}(>|Z|)$ \\
\hline $\begin{array}{c}\text { Intercepto fijo } \\
\text { Pendiente fija } \\
\text { Efecto } \\
\text { aleatorio }\end{array}$ & $\begin{array}{c}\text { Intercepto } \\
\text { aleatorio }\end{array}$ & & $\begin{array}{c}\text { Pendiente } \\
\text { Aleatoria }\end{array}$ & \\
\hline 2007 & $-7,801$ & & 0,761 & \\
2008 & $-15,106$ & & 1,193 & \\
2009 & $-8,956$ & & 0,866 & \\
2010 & $-3,548$ & & 0,425 & \\
2011 & $-24,463$ & & 2,258 & \\
2012 & $-25,018$ & & 2,403 & \\
\hline
\end{tabular}




\section{Discusión}

Las estimaciones de la talla de madurez $\left(L_{m}\right)$ de $S$. bentincki mostraron una variación interanual significativa en el periodo del 2007 al 2012, ya sea al utilizar modelo lineal generalizado (MLG) como modelo lineal generalizado de efectos mixtos (MLGM). Los cambios interanuales, sin embargo, no mostraron una tendencia ni a la disminución ni al aumento. Con ambas técnicas estadísticas, la talla de madurez más alta ocurrió en 2008 y la más baja en 2010. A excepción del 2008, los valores obtenidos fueron similares, en promedio, a lo encontrado por Arrizaga (1981), con $L_{m}=11 \mathrm{~cm}$; Mujica \& Rojas (1984), con $L_{m}=10 \mathrm{~cm}$; Arancibia et al. (1994), con $L_{m}=11 \mathrm{~cm}$; Cubillos et al. (1999), con $L_{m}=10 \mathrm{~cm}$. Cubillos et al. (2013) concluyeron que en la época reproductiva la estructura de tallas está caracterizada por ejemplares primerizos con moda entre $10-11 \mathrm{~cm}$ y otra moda en ejemplares repetitivos de $14-15 \mathrm{~cm}$ de longitud total. En el 2008 se registró la mayor incidencia de ejemplares inmaduros en peces más pequeños que $12 \mathrm{~cm}$ de longitud, lo que pudo determinar que la talla de madurez fuera más alta (13-15 cm). En 2010, el ajuste mostró que el 50\% de hembras maduras comienza a los $8,3 \mathrm{~cm}$; situación que no se advierte en los datos agrupados por clases de talla (Fig. 2).

Las estimaciones de $L_{m}$ presentaron la misma variación interanual con ambas técnicas estadísticas. Sin embargo, la diferencia metodológica entre MLG y MLGM, es que en el caso de MLG se asume independencia entre años. Esto implica que los cambios en la madurez con la talla exhiben variaciones interanuales independientes no relacionados, y por lo tanto la sardina común podría estar exhibiendo cambios en la talla de madurez que podrían deberse a condiciones específicas que se dan en cada año en particular. En cambio, el enfoque de estimación con MLGM asume que la talla de madurez es un proceso poblacional con parámetros fijos, y que las variaciones entre años con la talla se deben básicamente a efectos aleatorios que podrían ser debidos a varios factores asociados ya sea al muestreo (época y lugar), al crecimiento o a los retrasos en la madurez debido a la asincronía espacio-temporal del proceso. Desde el punto de vista de la evaluación de cambios en la talla de madurez que podrían estar asociados a un efecto de truncamiento de la estructura de tallas por efecto de la pesca, el enfoque de estimación con modelo mixto es más adecuado, ya que la talla de madurez no mostró una tendencia a disminuir como se podría esperar bajo la hipótesis de truncamiento de la estructura de tallas/edad (Anderson et al. 2008). Sin embargo, la variación aleatoria que se encontró en este estudio no puede ser atribuible a los efectos de la pesca sobre la fracción juvenil en forma concluyente.
Es factible que los cambios en la talla de madurez estén asociados a cambios en la magnitud del reclutamiento. En efecto, la sardina común presenta una fuerte dependencia de las capturas frente a las fluctuaciones que puede experimentar el reclutamiento. Cubillos et al. (2014) señala que una pesca intensa sobre juveniles genera un desplazamiento del máximo reproductivo estacional, lo que podría alterar la fenología reproductiva. De esta manera, la actividad pesquera que se desarrolla sobre los juveniles reclutados, antes que maduren, podría tener también consecuencias en la talla de primera madurez de sardina común. En efecto, mientras ocurre el reclutamiento se captura a los ejemplares juveniles de crecimiento rápido (más grandes) y de madurez temprana, seleccionando en la población ejemplares con crecimiento lento y madurez tardía. Sin embargo, los reclutamiento más abundantes que han ocurrido entre 2009 y 2012 (Castillo et al. 2013), podría estar enmascarando los efectos de la pesca en el periodo más reciente.

A diferencia de otros estudios en que la talla de madurez ha sido una estimación puntual para un año o periodo determinado (Arrizaga 1981, Mujica \& Rojas 1984, Arancibia et al. 1994, Cubillos et al. 1999), en este trabajo se estimó cambios en la talla de madurez de sardina común para una serie de 6 años. A pesar que la fracción inmadura comenzó a expresarse con una talla promedio menor entre 2011 y 2012, se observó que las variaciones interanuales de la talla de madurez se expresaron en valores por sobre y bajo las estimaciones conocidas (10-11 $\mathrm{cm}$ ), sin una tendencia a la disminución sistemática que implique cambios significativos en la talla de madurez que puedan ser atribuible a la pesca. De esta manera, las variaciones extremas ( 8.3 y $12,7 \mathrm{~cm}$ ) observadas deben ser analizadas considerando otros factores no considerados aquí, tal como los cambios interanuales en el crecimiento (e.g., Grover 2005, CastilloJordán et al. 2010) y que podrían determinar cambios en la talla de madurez. Se sugiere que futuros análisis consideren la evaluación de cambios simultáneos en la edad de madurez de la sardina común, única forma de saber si el crecimiento de las diferentes cohortes podría estar determinando cambios en la madurez de la sardina común en la zona centro-sur, o bien si se debe a los efectos del ambiente (e.g., Cardinale \& Modin 1999, Dominguez-Petit et al. 2008), o asincronía espacio-temporal en el ciclo reproductivo en la zona centro-sur.

\section{Agradecimientos}

Blanca Bustos agradece al Laboratorio de Evaluación de Poblaciones Marinas (EPOMAR), al proporcionar los elementos necesarios para la ejecución del presente estudio, 
además agradecer las observaciones realizadas por el Sr. Leonardo Castro (UdeC) y la Sra. Carolina Alarcón. Se agradece a COPAS Sur-Austral CONICYT PIA PFB31, por el financiamiento otorgado en la Beca COPAS Sur-Austral.

\section{LITERATURA CITADA}

Akaike H. 1987. Factor analysis and AIC. Psychometrika 56(3): 317-332.

Anderson CK, C-H Hsieh, S Sandin, R Hewitt, A Hollowed, J Beddington, RM May \& G Sugihara. 2008. Why fishing magnifies fluctuations in fish abundance. Nature 452: 835-839.

Arancibia H, L Cubillos, J Remmaggi \& R Alarcón. 1994. Determinación de la talla de primera madurez sexual y fecundidad parcial en la sardina común, Strangomera bentincki (Norman, 1936), del área de Talcahuano, Chile. Biología Pesquera 23: 11-17.

Arcos DF. 1987. Seasonal and short time scale variability in copepod abundance and species composition in an upwelling area off Concepción coast, Chile. Ph. D. Thesis, State University of New York, Stony Brook, 203 pp.

Arrizaga A. 1981. Nuevos antecedentes biológicos para la sardina común, Clupea (Strangomera) bentincki Norman 1936. Boletín de la Sociedad de Biología de Concepción, Chile 52: 5-66.

Bates D. 2010. Lme4: Mixed-effects modelling with R, 131 pp. Springer. <http://lme4.r-forge.r-project.org/lMMwR/ lrgprt.pdf>

Blaxter JHS \& JS Hunter. 1982. The biology of the clupeoid fishes. Advances in Marine Biology 20: 201-223.

Brown-Peterson N, D Wyanski, F Saborido-Rey, B Macewicz \& S Lowerre-Barbierie. 2011. A standardized terminology for describing reproductive development in fishes. Marine and Coastal Fisheries: Dynamics, Management, and Ecosystem Science 3: 52-70.

Buckland ST, KP Burnham \& NH Augustin. 1997. Model selection: An integral part of inference. Biometrics 53: 603618.

Cardinale M \& J Modin. 1999. Changes in size-at-maturity of Baltic cod (Gadus morhua) during a period of large variations in stock size and environmental conditions. Fisheries Research 41: 285-295.

Castillo-Jordán C, LA Cubillos \& E Navarro. 2010. Intercohort growth rate changes of common sardine (Strangomera bentincki) and their relationship with environmental conditions off central southern Chile. Fisheries Research 105: 228-236.

Castillo J, A Saavedra, V Catasti, F Leiva, C Lang, R Vargas, H Reyes, M Pizarro, E Molina, F Cerna, A López, S Nuñez, L Valenzuela \& J Silva. 2013. Evaluacioìn hidroacuística reclutamiento anchoveta sardina comuìn entre la V y X Regiones, año 2013. Informe Técnico FIP 2012-12: 1-307.<http://www.fip.cl/Archivos/Hitos/Informes/ INFORME\%20HITO\%20FINAL1104Adjunto1.pdf>
Claramunt G, LA Cubillos, L Castro, C Hernández \& M Arteaga. 2014. Variation in the spawning periods of Engraulis ringens and Strangomera bentincki off the coasts of Chile: a quantitative analysis. Fisheries Research 160: 96-102.

Cochran WG. 1980. Técnicas de muestreo, 513 pp. Compañía Editorial Continental, México.

Conover DO \& SB Munch. 2002. Sustaining fisheries yields over evolutionary time scales. Science 297: 94-96.

Cubillos L \& DF Arcos. 2002. Recruitment of common sardine (Strangomera bentincki) and anchovy (Engraulis ringens) off central-south Chile in the 1990s and the impact of the 1997-1998 El Niño. Aquatic Living Resources 15: 87-94.

Cubillos L, M Canales, A Hernández, D Bucarey, L Vilugrón \& L Miranda. 1998. Poder de pesca, esfuerzo de pesca y cambios estacionales e interanuales en la abundancia relativa de Strangomera bentincki y Engraulis ringens en el área frente a Talcahuano, Chile (1990-97). Investigaciones Marinas, Valparaíso 26: 3-14.

Cubillos L, M Canales, D Bucarey, A Rojas \& R Alarcón. 1999. Época reproductiva y talla media de primera madurez de Strangomera bentincki, y Engraulis ringens en el período 1993-1997 en la zona centro sur de Chile. Investigaciones Marinas, Valparaíso 27: 73-85.

Cubillos L, D Arcos, M Canales \& D Bucarey. 2001. Seasonal growth of small pelagic fish off Talcahuano $\left(37^{\circ} \mathrm{S}-73^{\circ} \mathrm{W}\right)$, Chile: a consequence of their reproductive strategy to seasonal upwelling? Aquatic Living Resources 14: 115-124.

Cubillos L, D Bucarey \& M Canales. 2002. Monthly abundance estimation for common sardine Strangomera bentincki and anchovy Engraulis ringens in the central-southern area off Chile (34- 40 $\mathrm{S}$ ). Fisheries Research 57: 117-130.

Cubillos L, P Ruiz, G Claramunt, S Gacitúa, S Núñez, L Castro, K Riquelme, C Alarcón, C Oyarzún \& A Sepúlveda. 2007. Spawning, daily egg production, and spawning stock biomass estimation for common sardine (Strangomera bentincki) and anchovy (Engraulis ringens) off central southern Chile in 2002. Fisheries Research 86: 228-240.

Cubillos LA, LR Castro, G Claramunt \& E Navarro. 2013. Evaluación del stock desovante de anchoveta y sardina común entre la V y X Regiones, año 2012. Informe Técnico FIP 2012-09: 1-96. <http://www.fip.cl/Archivos/Hitos/Informes/ INFORME\%20HITO\%20FINAL1076Adjunto1.pdf>

Cubillos LA, G Claramunt \& LR Castro. 2014. Simulation of fishery-induced changes on the reproductive cycle of common sardine, Strangomera bentincki, off central southern Chile. Fisheries Research 160: 103-111.

Dominguez-Petit R, M Korta, F Saborido-Rey, H Murua, M Sainza \& C Piñeiro. 2008. Changes in size at maturity of European hake Atlantic populations in relation with stock structure and environmental regimes. Journal of Marine Systems 71:260-278. 
Gatica C, M Arteaga, J Giacaman \& P Ruiz. 2007. Tendencias en la biomasa de sardina común (Strangomera bentincki) y anchoveta (Engraulis ringens) en la zona centro-sur de Chile, entre 1991 y 2005 . Investigaciones Marinas, Valparaíso 35(1): 3-12.

Grover MC. 2005. Changes in size and age at maturity in a population of kokanee Oncorhynchus nerka during a period of declining growth conditions. Journal of Fish Biology 66: 122-134.

Hunter JR \& BJ Macewicz. 1985. Measurement of spawning frequency in multiple spawning fishes. NOAA Technical Report, National Marine Fisheries Service 36: 79-94.

Mujica A \& O Rojas. 1984. Fecundidad y estructura poblacional de sardina común (Clupea bentincki, Norman) (Clupeiformes-Clupeidae). Investigación Pesquera 31: 5969.

Pinheiro J \& DM Bates. 2000. Mixed-Effects Models in S and S-PLUS, 528 pp. Springer (Statistics and Computing), New York.
R Core Team. 2014. R: A language and environment for statistical computing. R Foundation for Statistical Computing, Vienna. <https://cran.r-project.org/doc/manuals/r-release/ fullrefman.pdf $>$

Selman K \& R Wallace. 1989. Cellular aspects of oocyte growth in teleosts. Zoological Science 6: 211-231.

SERNAPESCA. 2013. Anuario estadístico de pesca 2013. Servicio Nacional de Pesca, Valparaíso. <http:// www.sernapesca.cl>

Vásquez P, A Llanos-Rivera \& L Castro. 2010. Anormalidades durante el desarrollo embrionario de sardina común, Strangomera bentincki en el ambiente natural. Revista de Biología Marina y Oceanografía 45(1): 177-185.

Venables WN \& BD Ripley. 2002. Modern applied statistics with S, 504 pp. Springer-Verlag, New York. <http://www.planta.cn/ forum/files_planta/modern_applied_statistics_with_s_192.pdf>

Wallace R \& K Selman. 1981. Cellular and dynamic aspects of oocyte growth in teleosts. American Zoologist 21: 325343

Recibido 19 de octubre 2015 y aceptado el 25 de marzo de 2016

Editor: Claudia Bustos D. 